\title{
Mesure des descendants du radon dans l'environnement du site du Bouchet
}

\author{
M.C. ROBÉ, V. LABED, A. BENEITO, J.M. MAUREL*
}

(Manuscrit reçu le 15 décembre 1994,
révisé le 25 août 1995, accepté le 14 septembre 1995)

RÉSUMÉ Le dépôt de résidus des fabrications d'uranium et de thorium qui ont eu lieu sur le site du Bouchet (Essonne) de 1949 à 1970 est une des composantes du système de traitement et de rejet des déchets de l'usine. Au cours des opérations de démantèlement et d'assainissement de cette ancienne usine de la Direction des productions du CEA 1 , la majeure partie des matières et matériels contaminés par des uranium et thorium ont été évacués (période de 1970-1979). Il subsiste deux zones occupant 1 ha : un bassin de décantation et une zone de dépôt pour les résidus solides. Les niveaux de flux d'émission radon avant la réhabilitation du site étaient $\mathbf{1 0}$ à $\mathbf{1 0 0}$ fois supérieurs à la moyenne des flux de radon à la surface de la terre $\left(10^{4}\right.$ atome $\left.m^{-2} s^{-1}\right)$ et les concentrations en descendants à vie courte du radon 222 étaient, dans l'environnement immédiat du site, supérieurs d'environ $20 \%$ aux niveaux prescrits en 1991 par un arrêté du préfet de l'Essonne. Les différentes mesures de radioactivité réalisées sur le site, ou dans son environnement proche, mettent en évidence l'efficacité de la réhabilitation réalisée en 1992-1993, les niveaux en concentration des descendants à vie courte du radon étant du même ordre de grandeur que ceux rencontrés dans le Bassin parisien.

ABSTRACT Between 1949 and 1971, the French Atomic energy commission operated a uranium ore processing facility at Le Bouchet, $\mathbf{4 0} \mathrm{km}$ from Paris. The plant was dismantled in the seventies. However, an additional patch of land, next door to the main premises had served for the disposal of uranium ore process mill tailings until 1956, and until 1971 as a settling basin for the plant effluent mud. The Bouchet plant operation has resulted in a deposit of tailings on a land area of $0.01 \mathrm{~km} 2$. The level of radon emissions from these premises before rehabilitation was 10 to 100 times greater than the mean level at the earth's surface, and the concentrations of radon decay products in the immediate environment of the site were on the average $20 \%$ above the levels legally allowed for the public in the vicinity of mines. The results obtained after this rehabilitation show that the short-lived radon decay products concentration has been lowered down to the same order of magnitude as the representative regional background noise in the Paris basin, typical of a sedimentary environment.

\footnotetext{
* Institut de protection et de sûreté nucléaire (IPSN), Département de protection de l'environnement et des installations, Service d'études et de recherches en aérocontamination et en confinement (DPEI/SERAC), CE/Saclay, Bâtiment 389, 91191 Gif-sur-Yvette Cedex.
}

1. CEA : Commissariat à l'énergie atomique 
M.C. ROBÉ et al.

\section{Introduction}

Le fonctionnement de l'usine de traitement de minerais, que le Commissariat à l'énergie atomique (CEA) a exploité au Bouchet jusqu'en 1970, a conduit à la constitution, sur un terrain annexe, d'un dépôt non couvert d'environ $20000 \mathrm{t}$ de résidus.

Comme pour les dépôts comparables qui sont associés à toutes les mines et usines de traitement des minerais d'uranium et de thorium, la nuisance principale est due à l'émission de radon (radon 220 et radon 222) provenant des radioéléments naturels présents dans les stériles et principalement les résidus. Les isotopes 220 et 222 du radon sont des gaz rares radioactifs de période courte, respectivement $55 \mathrm{~s}$ et $3,8 \mathrm{j}$. Leur désintégration entraîne l'apparition d'une succession de descendants solides; les premiers descendants sont euxmêmes radioactifs et leur présence dans l'air sous la forme d'aérosols inhalables constitue le risque sanitaire essentiel entraîné par l'émission de radon.

A partir de 1992, ce site a fait l'objet d'un réaménagement qui consistait à la mise en place d'une couverture constituée entre autres par une couche d'argile, dont il s'est avéré nécessaire de vérifier l'efficacité par des mesures de radon gaz et descendants.

\section{Le site du Bouchet et sa réhabilitation}

Entre 1949 et 1970, le CEA a exploité au Bouchet, sur la commune d'Itteville, dans l'Essonne, une installation de traitement de minerais d'uranium (Fig. 1). Après le démantèlement de cette installation, dans les années 70 , le terrain sur lequel elle était située a été rendu à son propriétaire, la SNPE (Société nationale des poudres et explosifs). Cependant, un terrain annexe, extérieur à l'installation, ayant servi jusqu'en 1956 au dépôt de résidus de traitement de minerais et, jusqu'en 1970, comme bassin de décantation des boues contenues dans les effluents de l'usine, est resté et demeure toujours sous la surveillance du CEA (Fig. 2). Les matériaux radioactifs contenus dans ce terrain proviennent donc exclusivement du traitement de minerais : ce sont des produits que l'on trouve, mais en proportions différentes, dans la nature (uranium, thorium, radium et leurs "descendants", comme le radon). Le fonctionnement de l'usine de traitement de minerais a donc conduit à la constitution, sur ce terrain annexe, d'un dépôt d'environ $20000 \mathrm{t}$ de résidus.

Dès 1990, différentes mesures ont été effectuées sur ce site et dans son environnement. En accord avec les autorités préfectorales et le CEA, l'Institut de protection de sûreté nucléaire (IPSN) a été chargé de réaliser :

- des mesures de radon gaz en continu sur de courtes durées afin de connaître très rapidement les niveaux de concentrations sur le site même et dans son environnement plus ou moins proche afin d'établir un diagnostic pour les autorités préfectorales ; 
- la cartographie des flux d'émission radon des terrains ;

- des mesures intégrées mensuelles des descendants à vie courte du radon (isotopes 220 et 222) sur de longues durées.

Ces dernières permettent une appréciation directe d'impact dosimétrique puisque celui-ci est estimé à partir de l'énergie alpha potentielle volumique $\left(E A P_{v}\right)^{1}$ qu'une personne pourrait absorber par inhalation en une année.

Un arrêté du préfet de l'Essonne (arrêté 91-1587 du 27 mai 1991) a fixé le cadre réglementaire de la surveillance de l'environnement pendant les années 1991 et 1992. Entre autres obligations, cet arrêté imposait au CEA "la mesure des niveaux de radioactivité dans l'environnement à l'extérieur du site et une cartographie de la radioactivité en radon à la surface du site des dépôts de déchets uranifères du CEA au Bouchet (commune d'Itteville, Essonne)".

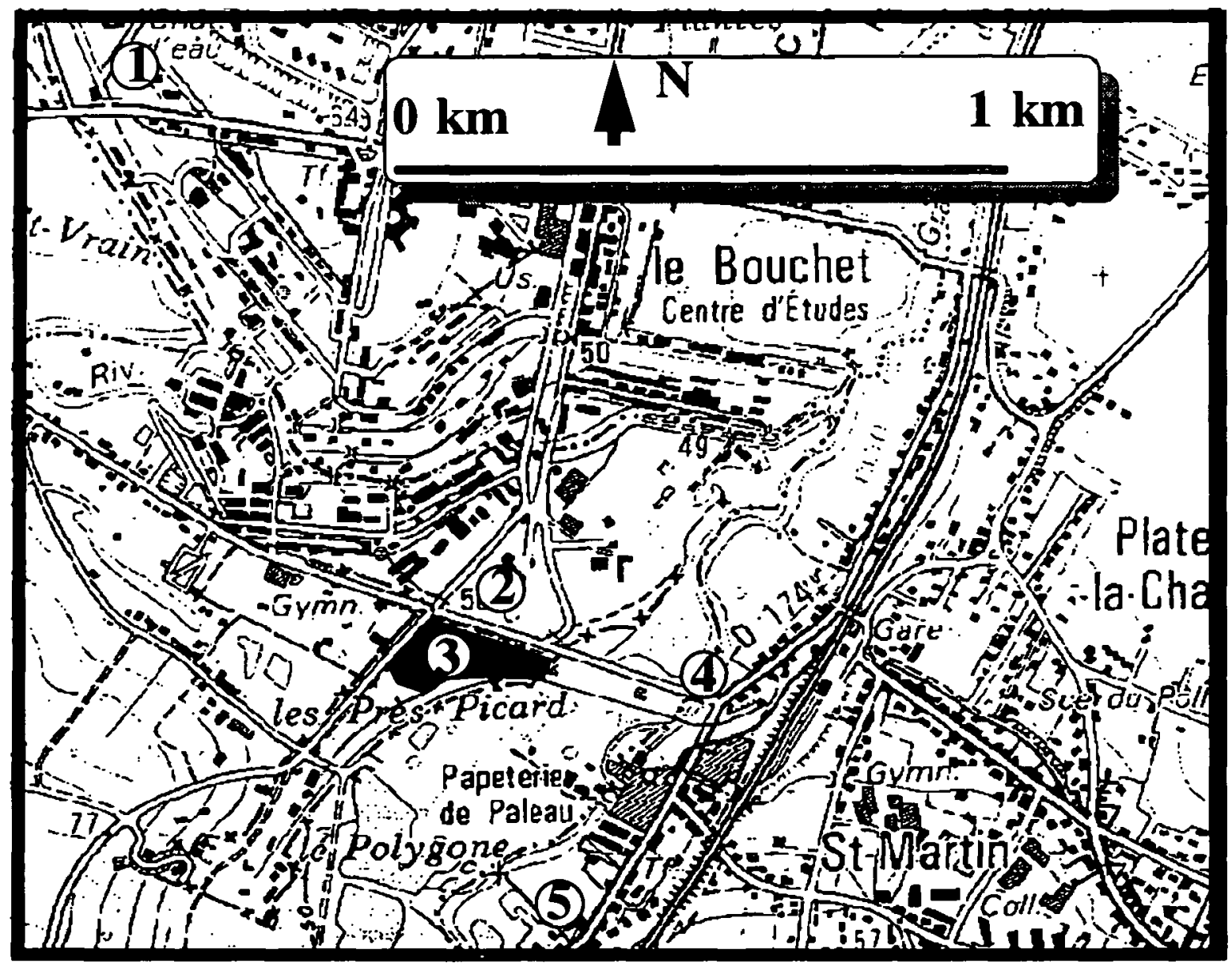

Fig. 1 - Emplacement des points de mesure. Location of the measurement points.

1. L'EAP $v$ est définie comme la somme des énergies des particules alpha émises au cours de la désintégration de tous les produits de filiation à vie courte du radon, contenus à un instant donné dans un certain volume d'air, pris comme unité. Ces mesures sont exprimées en nanojoule $\left(10^{-9} \mathrm{~J}\right)$ par mètre cube d'air $\left(\mathrm{nJ} . \mathrm{m}^{-3}\right)$. 


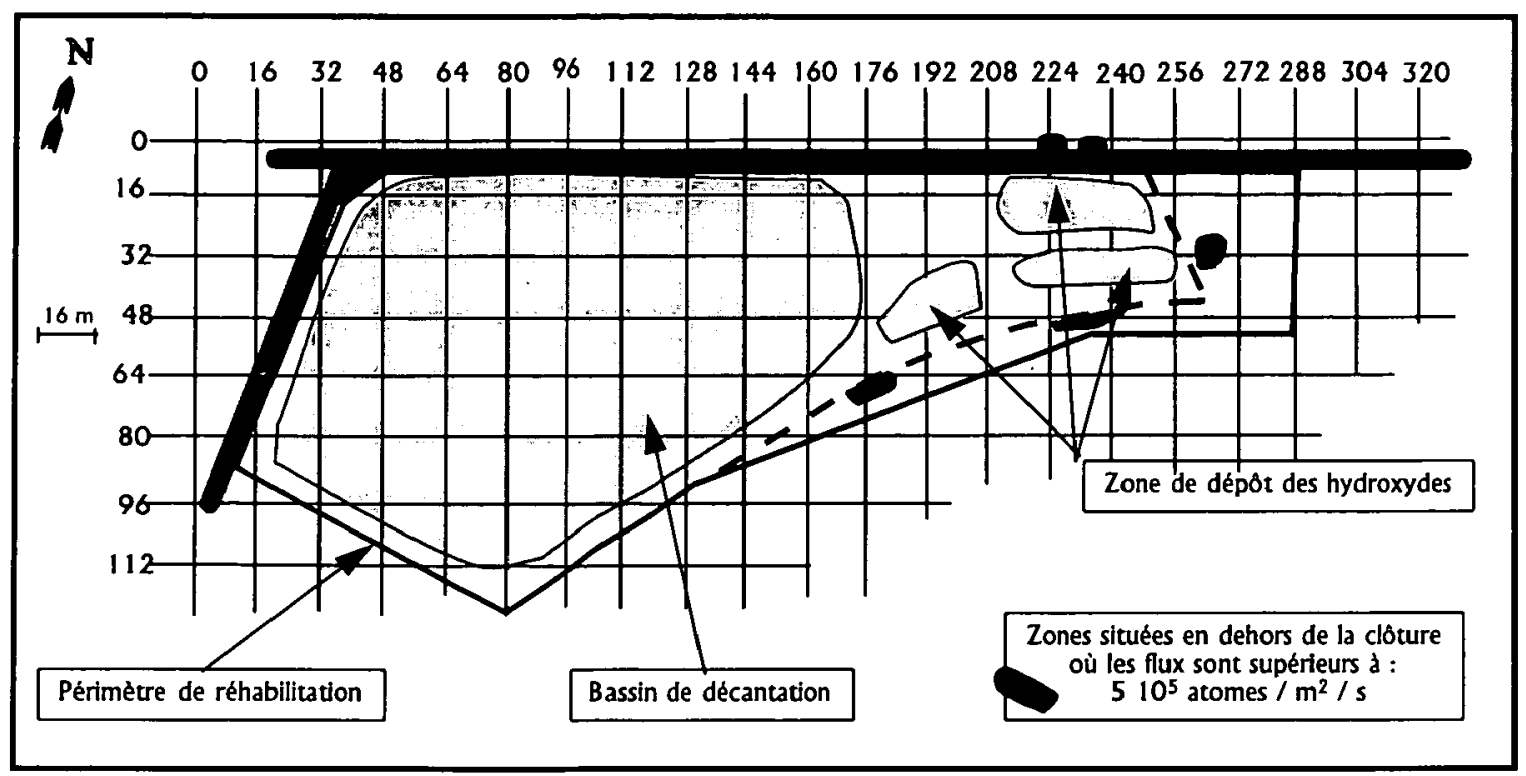

Fig. 2 - Site du Bouchet. The Bouchet site.

Puis, l'arrêté préfectoral n 92-2784 du 3 août 1992, qui a abrogé le précédent, a repris les mêmes dispositions dans le cadre du réaménagement du site (articles 2 et 5 ).

La mesure de l'EAP ${ }_{v}$ est effectuée à partir de cinq stations de prélèvement en continu. Leur implantation s'est faite en tenant compte des directions du vent, de l'orographie du site et de la présence du public (habitations proches du site).

Dès 1990, sur ces dépôts, des mesures ponctuelles dans le temps de radon gaz mettaient en évidence des niveaux dépassant $1000 \mathrm{~Bq} \cdot \mathrm{m}^{-3}$ en atmosphère libre soit des niveaux très supérieurs à ceux rencontrés en Ile-de-France (de l'ordre de quelques $\mathrm{Bq} . \mathrm{m}^{-3}$ sur des périodes de mesures similaires) [6]. Les mesures de flux d'émission radon ont mis en évidence des niveaux atteignant ponctuellement $7000 \cdot 10^{4}$ atomes $\mathrm{m}^{-2} \mathrm{~s}^{-1}$ soit plus de 5000 fois la valeur des flux habituellement observés dans la région. De même, les mesures mensuelles intégrées des descendants à vie courte du radon montraient des niveaux jusqu'à dix fois plus élevés que ceux habituellement observés en région parisienne et de ce fait trop importants dans l'environnement immédiat du dépôt, du moins dans l'hypothèse de l'exposition d'une personne qui passerait une année entière $(8760 \mathrm{~h})$ sur le terrain ou à sa proximité immédiate. A quelques centaines de mètres, cette concentration chutait au niveau naturel correspondant au bruit de fond régional.

L'article 2 de l'arrêté préfectoral du 3 août 1992 fixe pour la réhabilitation les objectifs à atteindre $[2,3]$ : "les travaux de réhabilitation, et notamment l'épaisseur de la couche d'argile à mettre en place, doivent permettre de respecter 
dans l'atmosphère, au droit du dépôt, les limites de concentration moyenne annuelle en énergie alpha potentielle fixées comme suit:

- 280 nJ.m-3 pour les descendants à vie courte du radon 222,

- $850 \mathrm{~nJ} . \mathrm{m}^{-3}$ pour les descendants à vie courte du radon 220.

Les limites s'expriment en valeur ajoutée aux concentrations naturelles en région parisienne soit respectivement 40 et $30 \mathrm{~nJ} . \mathrm{m}^{-3 "}$.

Il est prévu qu'après cette réhabilitation le site reste inconstructible.

Les travaux de réhabilitation ont débuté au cours du second semestre 1992. Ils se sont déroulés en 4 phases :

Phase 1 : travaux préliminaires (fin 1992). Au cours de cette phase, le chantier a été mis en place, le terrain débroussaillé et les ferrailles nettoyées à l'eau sous pression et contrôlées avant d'être évacuées.

Phase 2 : terrassement (1 $1^{\mathrm{er}}$ trimestre 1993). Il a consisté à aplanir et à mettre en forme le terrain (compactage) puis à mettre en place un treillis métallique et une membrane géotextile pour séparer les résidus de traitement de la couche d'argile.

Phase 3 : pose de l'argile $\left(2^{\mathrm{e}}\right.$ trimestre 1993). Une couverture d'argile présélectionnée par tests en laboratoire, de plusieurs dizaines de centimètres d'épaisseur, a été mise en place puis compactée.

Phase 4 : recouvrement et finition (4e trimestre 1993). Une couche drainante constituée de sable et de graviers maintient l'argile humide et fait obstacle à la pénétration d'animaux. L'ensemble du terrain a été couvert de terre végétale et de gazon.

\section{Localisation des points de mesure de l'EAP,}

La mesure de l'EAP ${ }_{v}$ est effectuée à partir de cinq stations. La localisation des points de mesure est présentée dans le tableau I et la figure 1.

La station 1 , située à $1,2 \mathrm{~km}$ du site du Bouchet et au vent de celui-ci, est considérée comme représentant l'exposition naturelle de cette région.

\section{Résultats}

\subsection{Mesure de flux d'émission radon}

Les distributions en fréquence des flux d'émission radon 222 mesurés à l'interface sol-atmosphère [4] avant toute réhabilitation en 1991 et de ceux mesurés après mise en place de la couche d'argile mettent en évidence l'efficacité des réaménagements (Fig. 3). Rappelons que le flux moyen à la surface de la terre est de $10^{4}$ atomes $\mathrm{m}^{-2} \mathrm{~s}^{-1}$. Avant le réaménagement, les flux étaient 
pour $75 \%$ compris entre $10^{4}$ et $10^{9}$ atomes $\mathrm{m}^{-2} \mathrm{~s}^{-1}$. Après travaux, $68 \%$ des mesures étaient inférieures à $10^{4}$ atomes $\mathrm{m}^{-2} \mathrm{~s}^{-1}$.

TABLEAU 1

Localisation des cinq stations de prélèvement Location of the five sampling stations

\begin{tabular}{|c|c|c|}
\hline \multicolumn{3}{|c|}{ 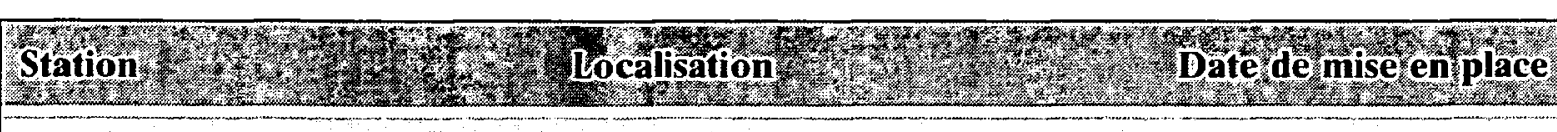 } \\
\hline 1 & Château d'eau de Vert le Petit, hors influence du site & 6 février 1990 \\
\hline 2 & En limite de site, au nord du dépôt & 6 février 1990 \\
\hline 3 & En limite de site, à l'ouest du bassin de décantation & 17. septembre 1990 \\
\hline $3(1)$ & Sur le dépôt & $1^{\text {er }}$ mars 1994 \\
\hline 4 & $\begin{array}{l}\text { A Ballancourt, dans l'environnement du site, } \\
\text { à l'est du dépôt }\end{array}$ & 17 septembre 1990 \\
\hline 5 & $\begin{array}{l}\text { A Ballancourt, dans l'environnement du site } \\
\text { au sud-est du dépôt }\end{array}$ & \\
\hline
\end{tabular}

(1) la station 3, conformément à un arrêté préfectoral du 18 février 1994 a été déplacée en un point central du site en raison de problèmes d'alimentation électrique.

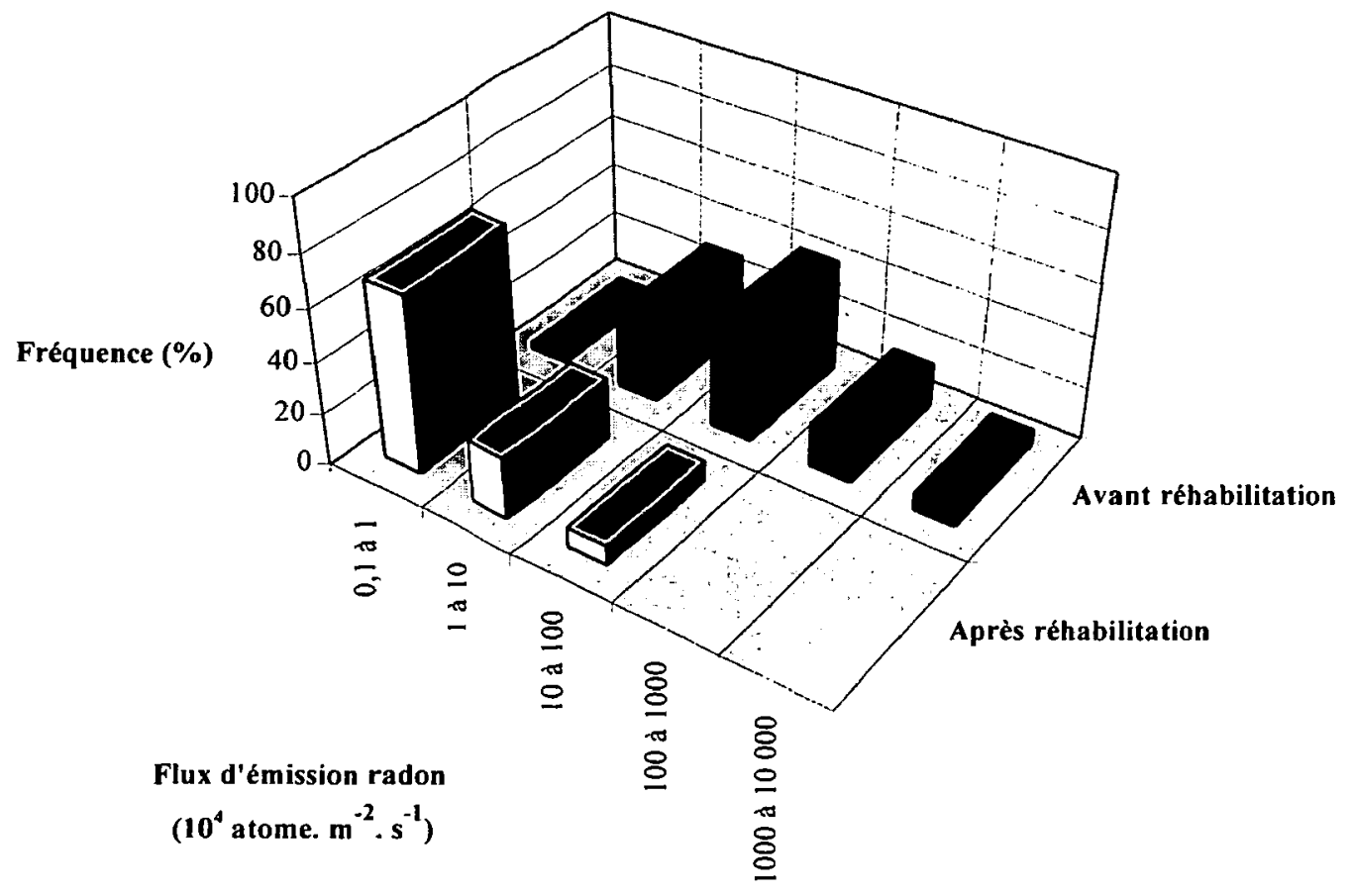

Fig. 3 - Distribution en fréquence des flux d'émission radon mesurée sur le site du Bouchet avant sa réhabilitation et après la mise en place de la couche d'argile. Flux frequency distribution before rehabilitation and after installing a clay cover. 


\subsection{Mesure de l'EAP}

On trouvera dans le tableau II les valeurs moyennes annuelles d'EAP $\mathrm{v}_{\mathrm{v}}$ pour chaque station de mesure. L'incertitude de mesure est de $20 \%$.

\section{TABLEAU II}

Energie alpha potentielle volumique $\left(\mathrm{nJ} / \mathrm{m}^{3}\right)$ due aux descendants à vie courte du radon. Moyenne annuelle des mesures effectuées de 1990 à 1994

$\mathrm{PAE}_{\mathrm{v}}\left(\mathrm{nJ} . \mathrm{m}^{-3}\right)$ due to the short-lived radon decay products.

Annual mean of the measurements carried out from 1990 to 1994

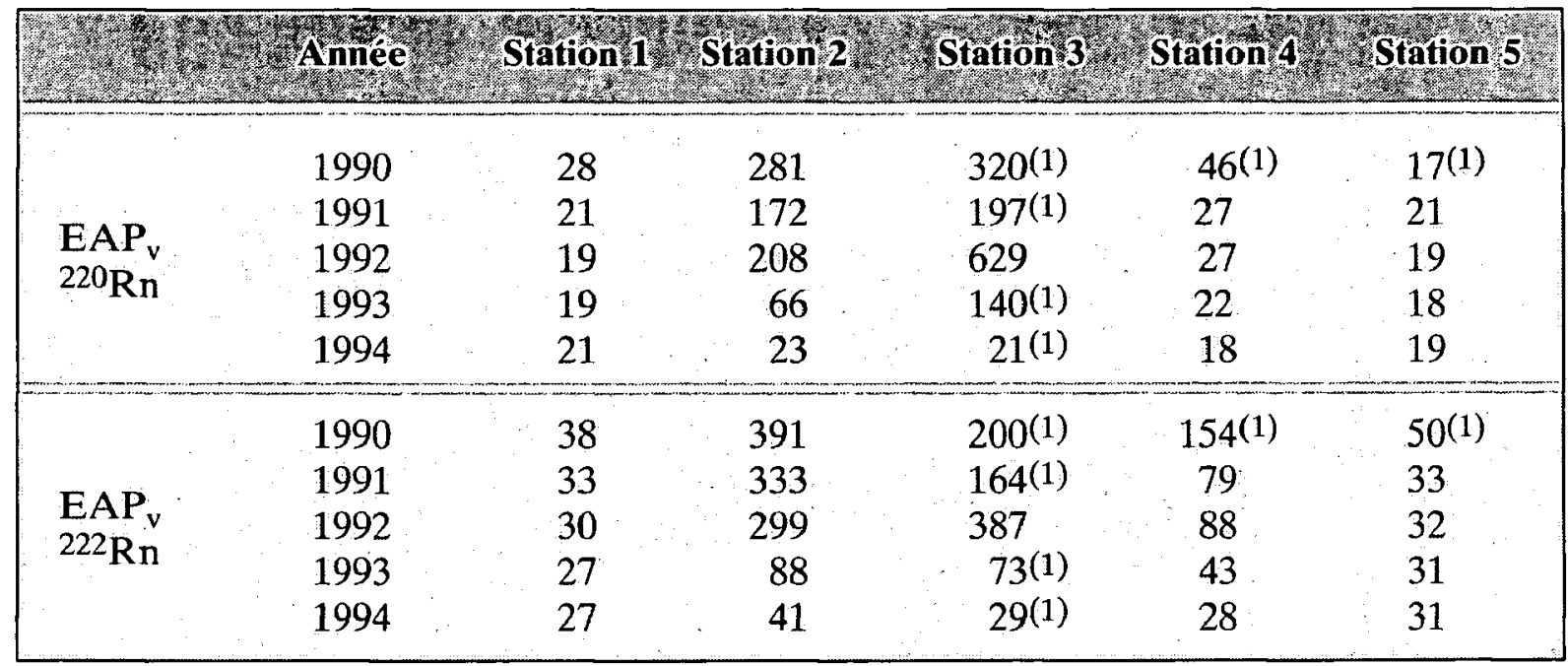

(1) estimation.

Remarque : l'estimation de la moyenne annuelle (Tab. II) est d'autant moins fiable que la durée de la mesure a été courte : ainsi, pour les stations 3, 4 et 5, l'estimation de la situation en 1990 est approximative. Notons que pour 1991, 1993 et 1994, l'estimation reste approximative uniquement pour la station 3 , en raison d'arrêts occasionnels du fonctionnement de cette station.

\section{Stations 1 et 5 (Figs. 4 et 5)}

Les représentations graphiques des variations de l'EAP pour les isotopes 220 et 222 des stations 1 et 5 mettent en évidence que les niveaux mesurés sur ces deux stations ne sont pas significativement différents. On notera dans le tableau III les valeurs moyennes sur près de 5 ans de mesures, qui peuvent être considérées comme représentatives du bruit de fond régional. Cette hypothèse a été confirmée par les résultats des mesures d'EAP $_{\mathrm{v}}$ réalisées à une trentaine de kilomètres du site, dans un contexte géologique semblable, où des niveaux de 30 et $22 \mathrm{~nJ} . \mathrm{m}^{-3}$ ont été observés sur quelques mois pour les descendants des isotopes 222 et 220 . Rappelons que l'arrêté préfectoral retient 30 et $40 \mathrm{~nJ} . \mathrm{m}^{-3}$ comme valeurs moyennes de la concentration naturelle en région parisienne pour les descendants à vie courte des isotopes 220 et 222 du radon, respectivement. 
M.C. ROBÉ el al.
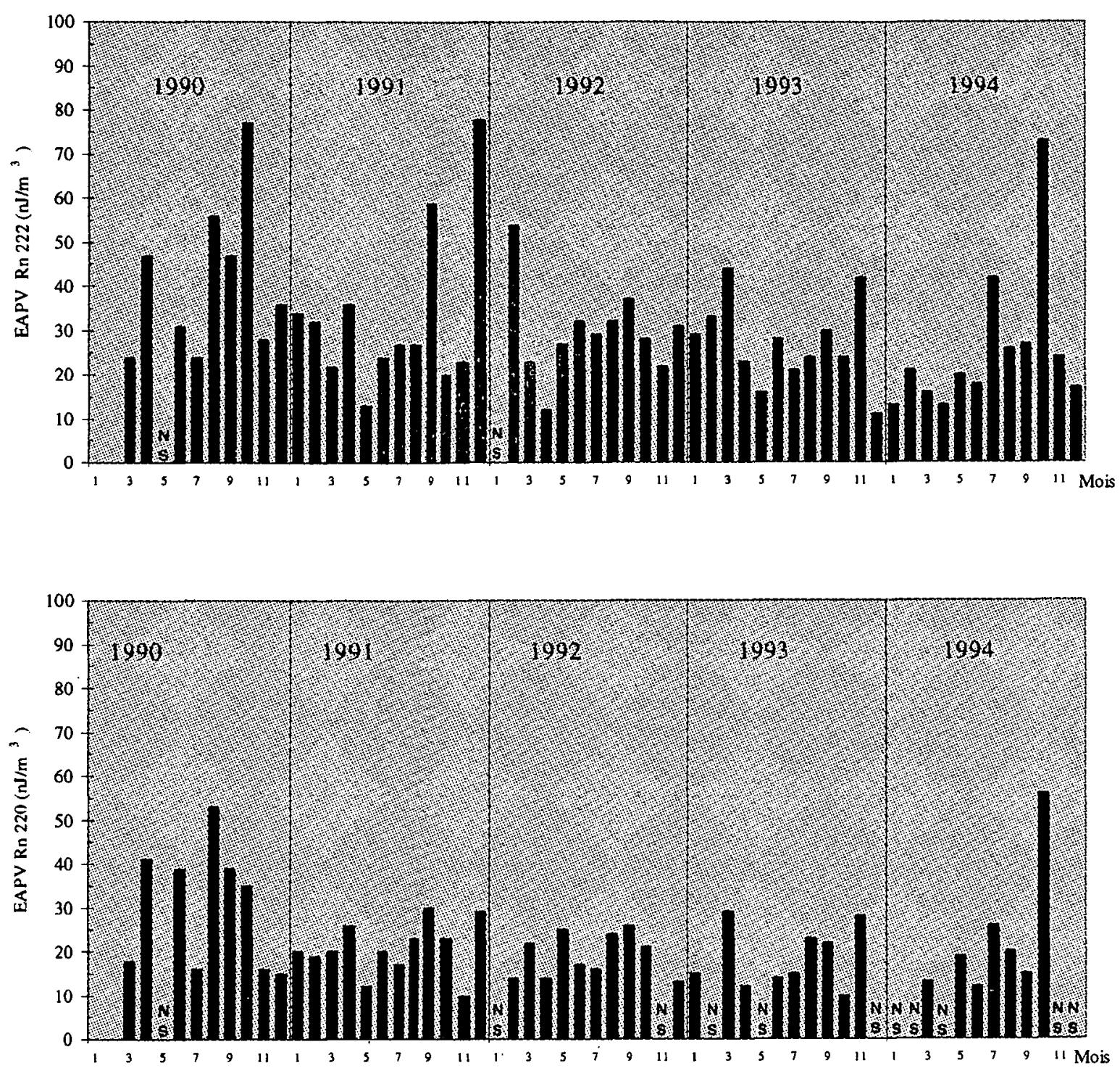

Fig. 4- EAP ${ }^{220}$ Rn et EAP ${ }^{222} R n$ dans l'environnement plus ou moins éloigné du site du Bouchet. La station 1 est située au vent du site.

${ }^{220} R n$ PAE and ${ }^{222} R n$ PAE. The station 1 is characteristic of the background noise in the region and is located upwind the site.

$N S=$ non significatif $\left(<10 \mathrm{~nJ} . \mathrm{m}^{-3}\right)$. 
MESURE DES DESCENDANTS DU RADON
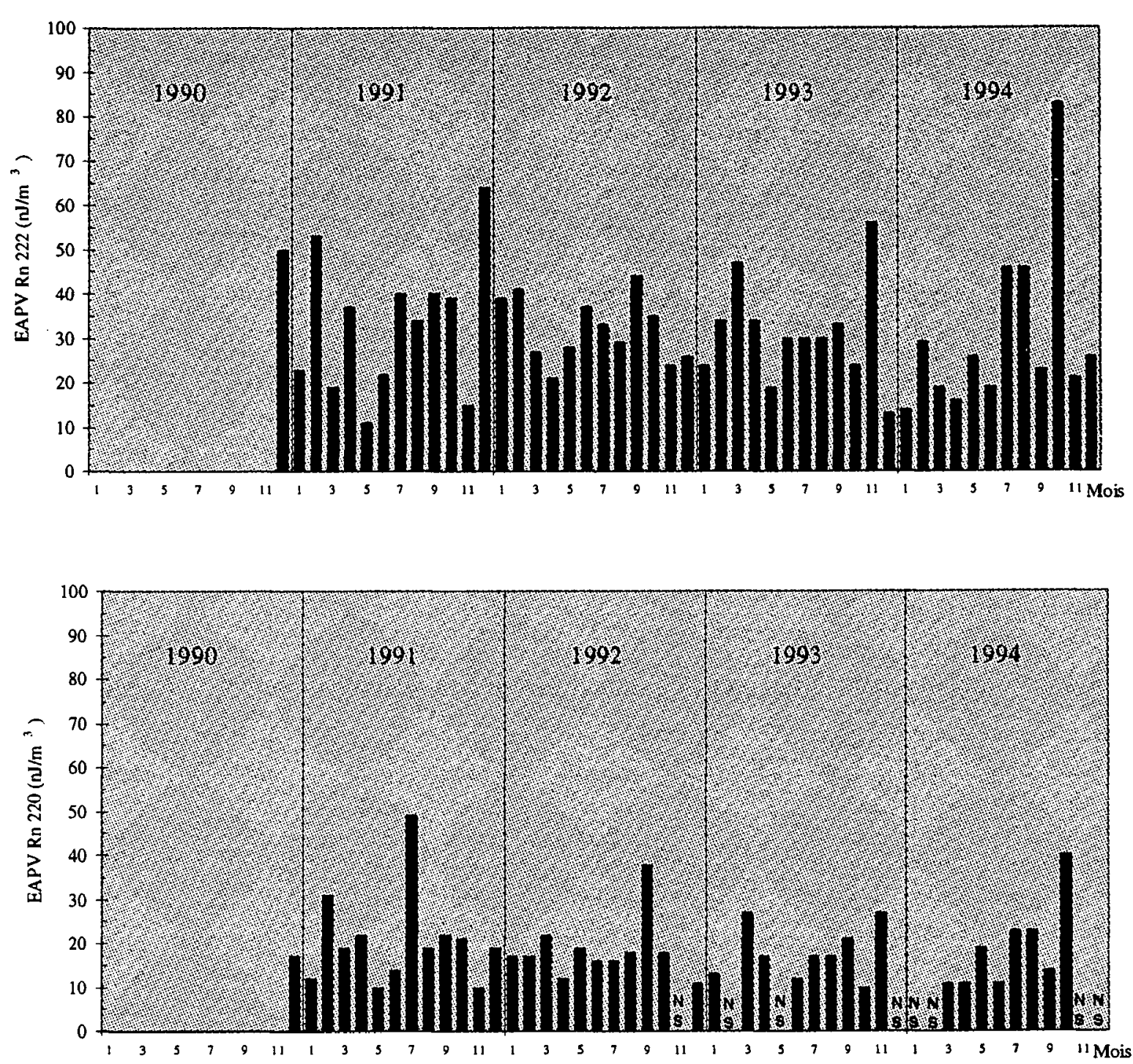

Fig. 5 - EAP ${ }^{220} R$ n et EAP ${ }^{222}$ Rn dans l'environnement plus ou moins éloigné du site du Bouchet (station 5).

${ }^{220} \mathrm{Rn} P A E$ and ${ }^{222} \mathrm{Rn} P A E$ in the vicinity of the site or far from it (station 5). $N S=$ non significatif $\left(<10 \mathrm{~nJ} . \mathrm{m}^{-3}\right)$.

TABLEAU III

Concentrations moyennes en énergie alpha potentielle volumique $\left(\mathbf{n J . m}^{-3}\right)$ sur près de 5 ans de mesure, pour les stations 1 et 5

PAE $E_{v}$ mean concentrations $\left(n J . m^{-3}\right)$ over 5 years, stations 1 and 5

\begin{tabular}{|c|c|c|}
\hline bent? & $\mathbf{E A P}_{\mathbf{Y}} 220 \mathrm{Rn}$ & $\mathbf{E A P}_{\mathbf{v}}{ }^{222} \mathbf{R n}$ \\
\hline Station 1 & 22 & 31 \\
\hline Station 5 & 19 & 32 \\
\hline
\end{tabular}




\section{Station 4 (Fig. 6)}

Pour l'isotope 222, on note une légère diminution des niveaux d'EAP au cours de l'année 1993, et plus particulièrement après les travaux d'aplanissement du terrain.
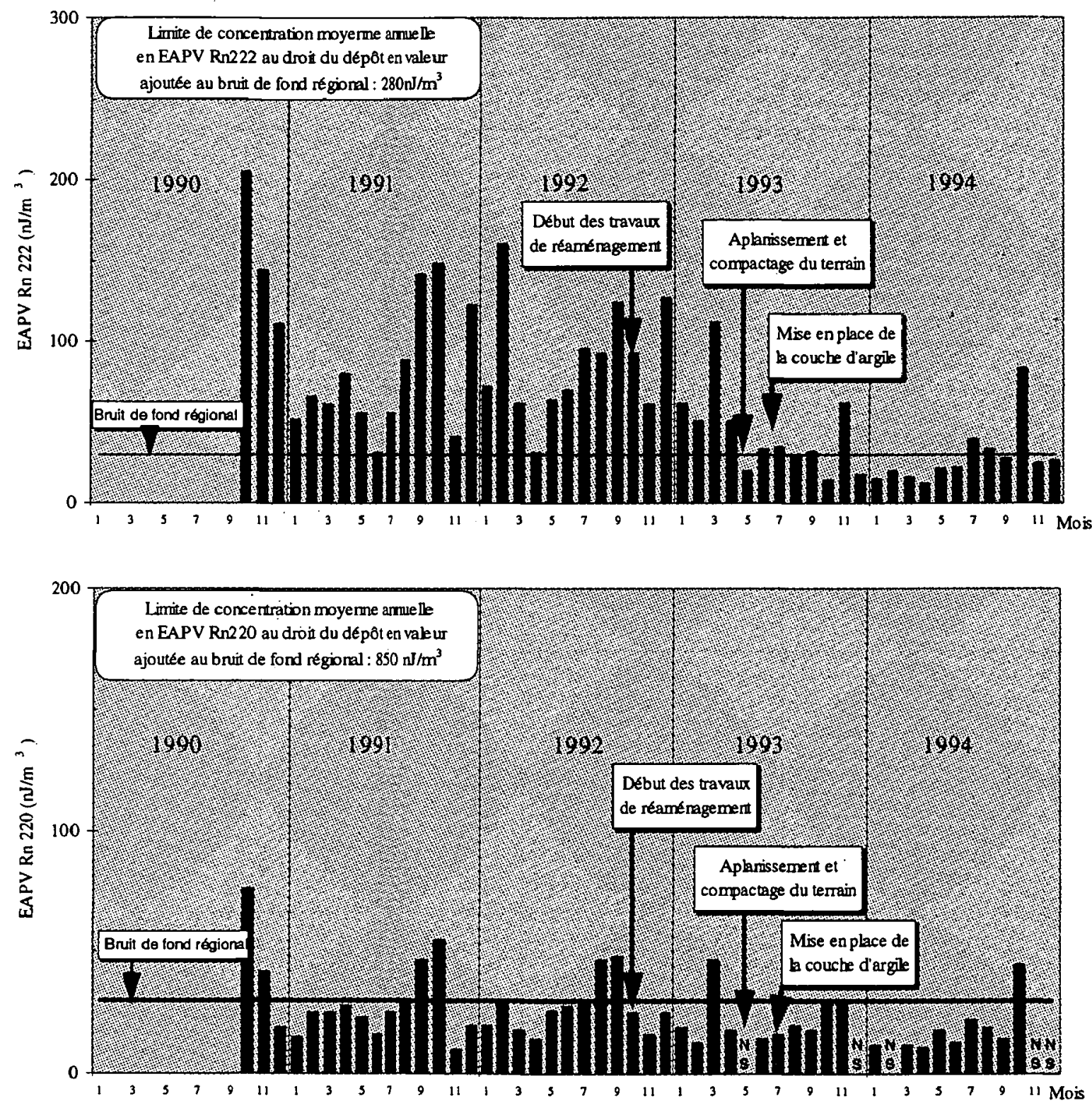

Fig. 6 - EAP ${ }^{220}$ Rn et EAP ${ }^{222} R n$ dans l'environnement plus ou moins éloigné du site du Bouchet (station 4).

${ }^{220} R n P A E$ and ${ }^{222} R n P A E$ in the vicinity of the site or far from it (station 4). $N S$ : non significatif $\left(<10 \mathrm{~nJ} . \mathrm{m}^{-3}\right)$. 
Stations 2 et 3 (Figs. 7 et 8 )

- Avant les travaux de réhabilitation et jusqu'au début des travaux de réhabilitation
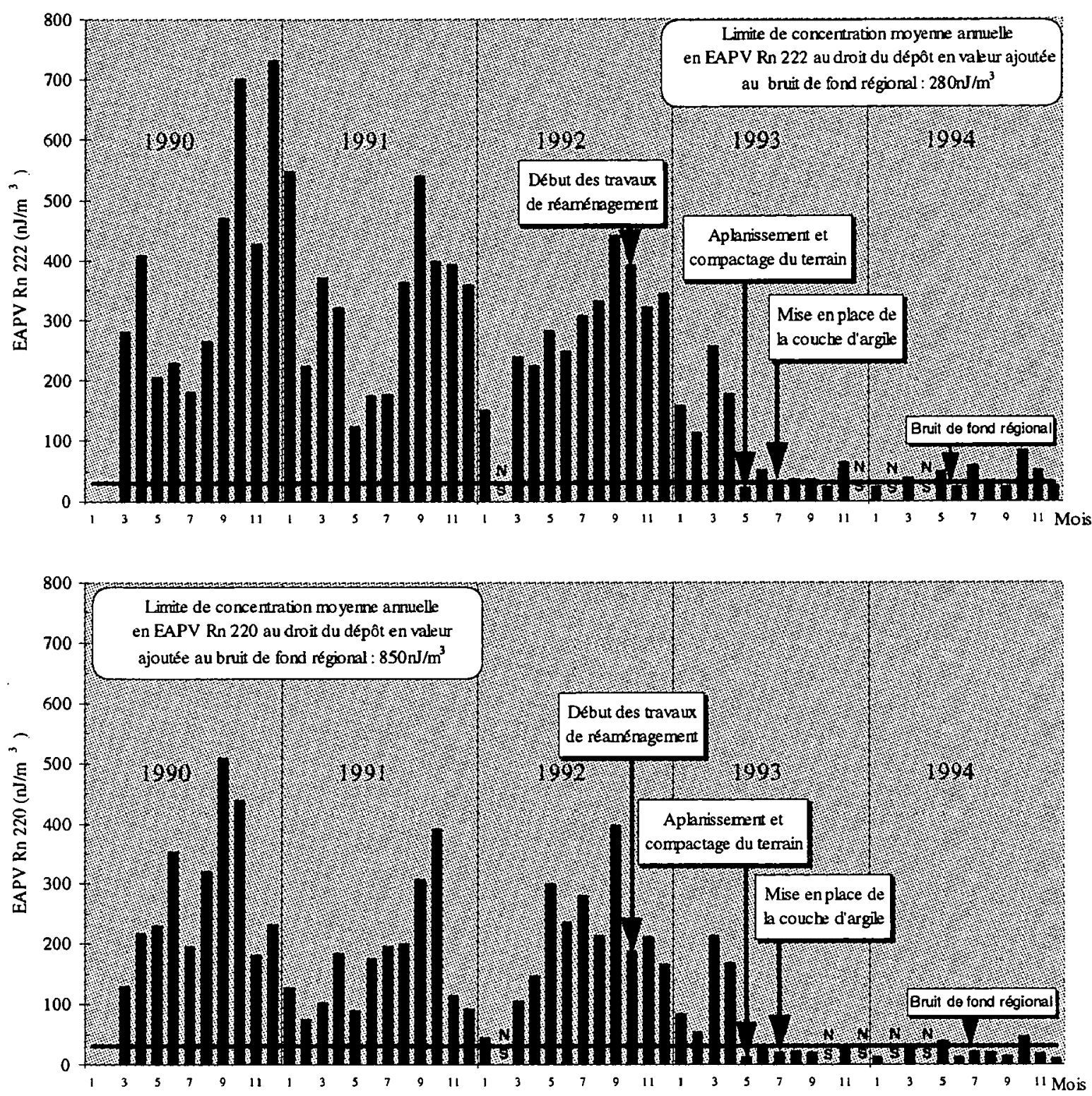

Fig. 7 - EAP ${ }^{220} R n$ et EAP ${ }^{222} R n$ dans l'environnement très proche du site du Bouchet (station 2).

${ }^{220}$ Rn PAE and $222 \mathrm{Rn} P A E$ in the nearest surrounding og the disposal (station 5). $N S$ : non significatif $\left(<10 \mathrm{~nJ} . \mathrm{m}^{-3}\right)$.

A ces 2 stations, les niveaux relevés tant pour l'isotope 220 que pour l'isotope 222 présentent une grande variabilité. Une première analyse de ces résultats met en évidence une évolution similaire d'une année sur l'autre, à savoir : une diminution des concentrations en fin d'hiver, début de printemps et une augmentation notable aux mois d'août et septembre. 


\section{- Après les travaux de réhabilitation}

A partir du mois de mai 1993, soit après la phase d'aplanissement et compactage du terrain, les niveaux $\mathrm{d}^{\prime} \mathrm{EAP}_{\mathrm{v}}$ tendent vers les valeurs représentatives du bruit de fond régional (Tab. IV).
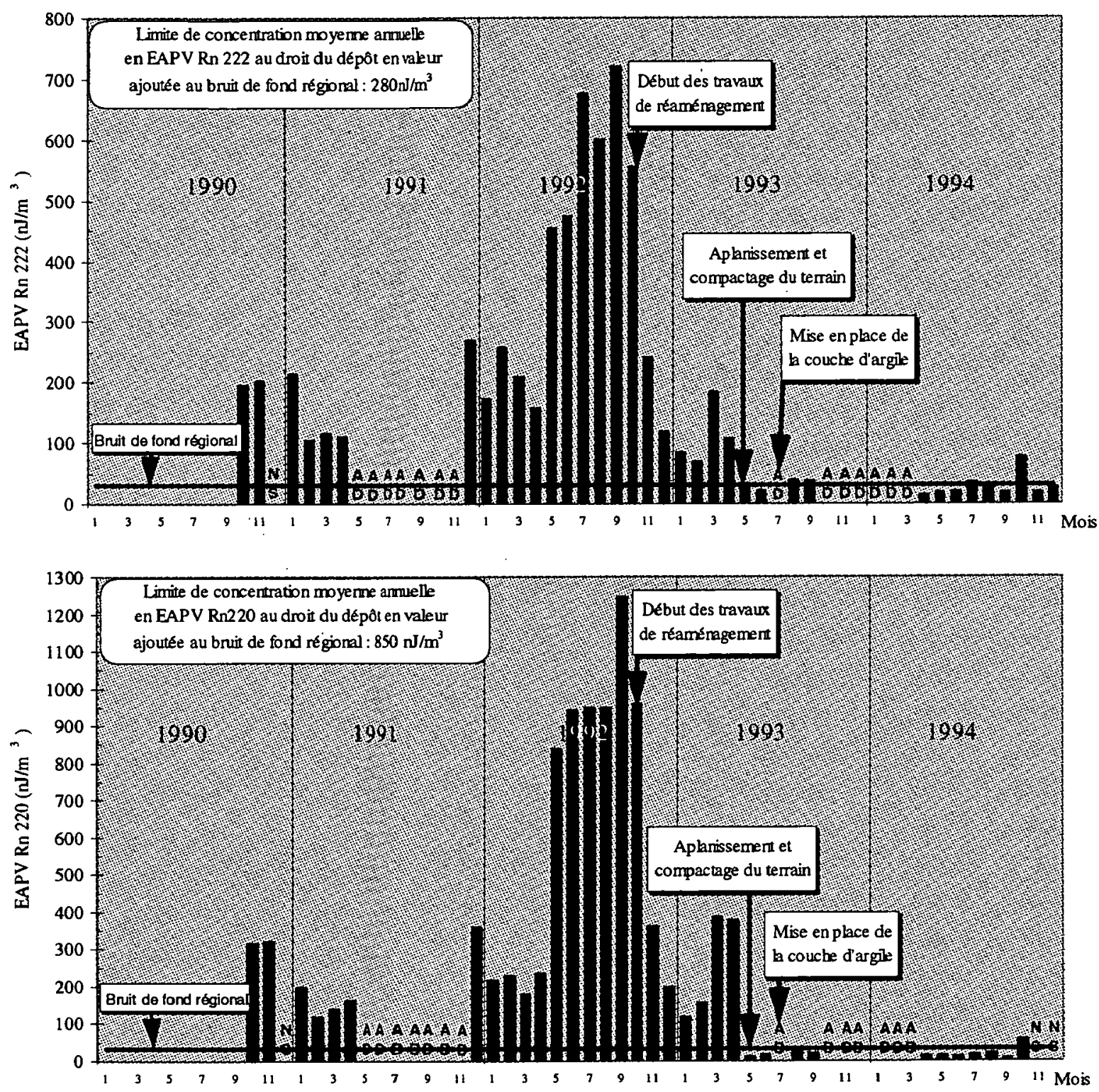

Fig. 8 - EAP ${ }^{220}$ Rn et EAP ${ }^{222} R n$ à la clôture du site (station 3). Après les travaux de réhabilitation, la station 3 est sur le site.

${ }^{220}{ }_{R n}$ PAE and ${ }^{222} R n$ PAE at the enclosure of the site (station 3). After the end of the rehabiliation, the station 3 is above the disposal.

$N S$ : non significatif $\left(<10 \mathrm{~nJ} \cdot \mathrm{m}^{-3}\right) ; A D$ : appareil débranché. 


\section{Interprétation}

Pour calculer l'énergie alpha potentielle absorbée par inhalation par une personne, il faut connaître la fraction de l'année pendant laquelle elle a séjourné au lieu considéré et le volume d'air qu'elle a inhalé.

TABLEAU IV

Concentrations moyennes en énergie alpha potentielle volumique (nJ $\left.\mathbf{m}-{ }^{3}\right)$

de mai à décembre 1993 (soit après la phase de compactage du terrain) et pendant l'année 1994 (soit sur le site réaménagé)

PAE $E_{v}$ mean concentration (nJ.m-3), May-December 1993 (after ground compaction) and in 1994 (on the rehabilitated site)

\begin{tabular}{|c|c|c|c|}
\hline \multicolumn{4}{|c|}{ 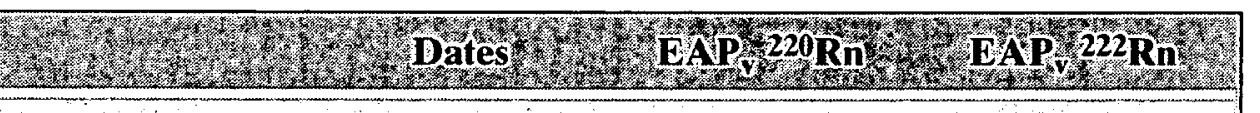 } \\
\hline Station 2 & $\begin{array}{l}05 / 93-12 / 93 \\
01 / 94-12 / 94\end{array}$ & $\begin{array}{l}23 \\
23\end{array}$ & 38 \\
\hline Station 3 & $\begin{array}{l}05 / 93-12 / 93 \\
01 / 94-12 / 94\end{array}$ & $\begin{array}{l}33(1) \\
28\end{array}$ & $\begin{array}{l}21^{(1)} \\
31\end{array}$ \\
\hline
\end{tabular}

(1) estimation.

Les réglementations nationale et internationale fixent des "limites annuelles d'incorporation" (LAI), en valeur ajoutée à l'exposition naturelle, pour les travailleurs et pour le public inhalant de l'air contenant du radon :

- LAI travailleurs : $20 \mathrm{~mJ} / \mathrm{an}$ en ${ }^{222} \mathrm{Rn}, 60 \mathrm{~mJ} / \mathrm{an}$ en ${ }^{220} \mathrm{Rn}$,

- LAI public : $2 \mathrm{~mJ} / \mathrm{an}$ en ${ }^{222} \mathrm{Rn}, 6 \mathrm{~mJ} / \mathrm{an}$ en ${ }^{220} \mathrm{Rn}$.

Les débits d'inhalation estimés pour l'homme standard sont :

- travailleurs : $1,2 \mathrm{~m}^{3} / \mathrm{h}\left(10 \mathrm{~m}^{3}\right.$ pour un poste de travail de $\left.8 \mathrm{~h}\right)$,

- personnes adultes du public, moyenne sur $24 \mathrm{~h}: 0,8 \mathrm{~m}^{3} / \mathrm{h}\left(20 \mathrm{~m}^{3}\right.$ par jour $)$.

Rappelons que l'arrêté préfectoral du 3 août 1992 fixe, dans l'article 2, comme objectif à atteindre après travaux de réaménagement des limites de concentrations moyennes annuelles en $\operatorname{EAP}_{\mathrm{v}}$, au droit du dépôt en valeurs ajoutées aux concentrations naturelles :

$-280 \mathrm{~nJ} / \mathrm{m}^{3}$, pour les descendants à vie courte $d u^{222} R \mathrm{n}$,

$-850 \mathrm{~nJ} / \mathrm{m}^{3}$, pour les descendants à vie courte $d u^{220} \mathrm{Rn}$.

Ces limites sont déterminées en considérant la LAI public et le débit d'inhalation de $0,8 \mathrm{~m}^{3} / \mathrm{h}$ sur une base annuelle de $8760 \mathrm{~h}$. Bien entendu, la règle fixée par l'arrêté préfectoral est extrêmement "conservative" puisqu'il est manifeste qu'aucune hypothèse réaliste ne pourrait conduire une personne à séjourner, sur le site même, 24 h par jour, toute l'année. 
Malgré le caractère discutable de cette hypothèse, il est possible de calculer la fraction de LAI apportée par le dépôt pour une personne qui aurait séjourné en permanence $(8760 \mathrm{~h})$ à l'emplacement des stations de mesure en s'appuyant sur les considérations suivantes :

- on suppose que la station 1 représente, compte tenu de son éloignement du site du Bouchet, l'exposition naturelle de cette région, et donc celle qui aurait été mesurée aux différentes stations en l'absence de dépôt ;

- la différence entre les résultats des mesures obtenues sur la station 1 , et ceux de chacune des stations 2, 3, 4, 5 permet de déterminer l'augmentation de l'EAP attribuable au dépôt ;

- on rapporte les chiffres précédents à la LAI "public".

D'où les résultats exprimés en \% de LAI dans le tableau V. Les différents calculs sont faits en considérant la référence naturelle (station 1) propre à chaque année.

TABLEAU $\mathrm{V}$

Augmentation de l'exposition du public, en pourcentage des limites annuelles d'incorporation par rapport à la référence naturelle Increase of public exposure in percent of ALIS vs natural exposure

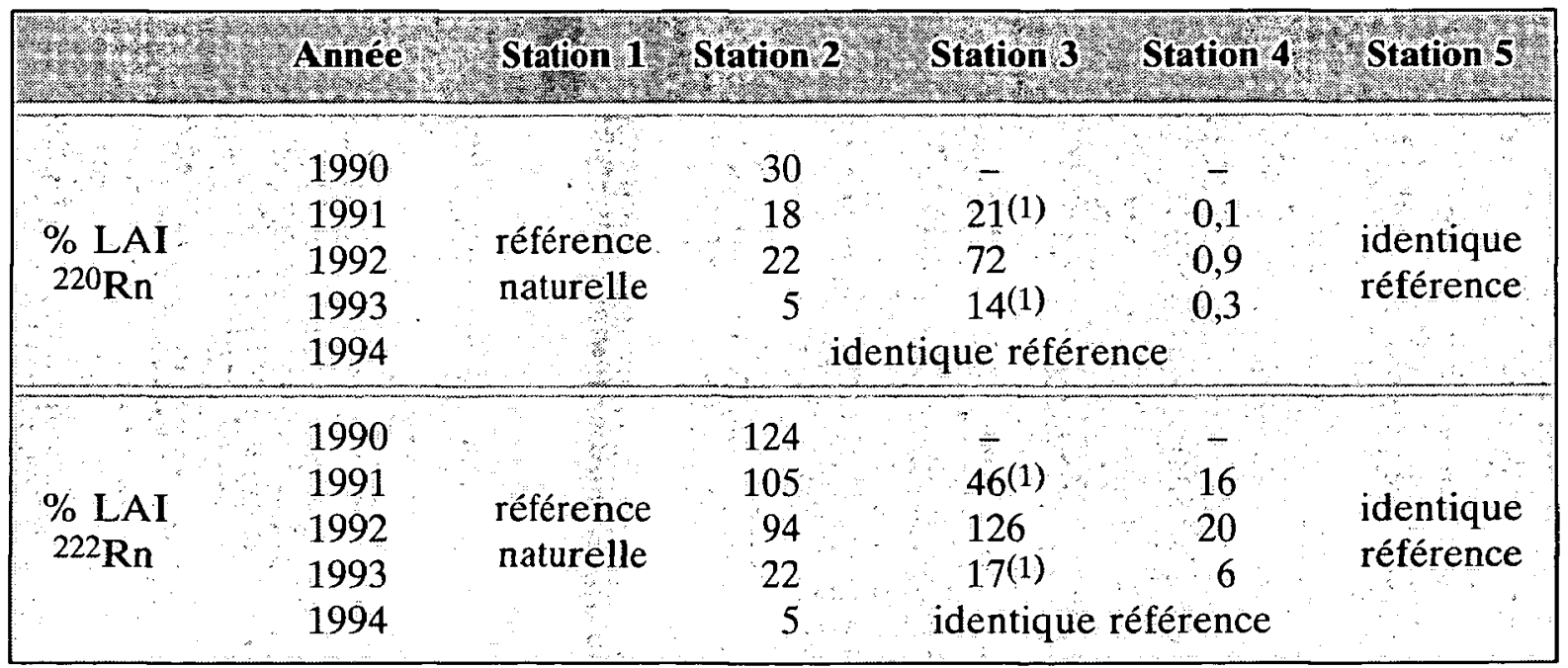

(1) estimation.

Pour l'année 1990, on ne peut raisonnablement procéder à ce calcul que pour la station 2 .

\section{Conclusions}

Les mesure intégrées mensuelles effectuées de 1990 à 1994 sur cinq stations du site du Bouchet permettent d'estimer l'énergie alpha potentielle volumique $\left(E A P_{v}\right)$ des descendants à vie courte du radon sur les différents emplacements. 
Le risque dosimétrique potentiel associé peut être apprécié au travers du calcul du pourcentage de la limite annuelle d'incorporation pour une personne du public séjournant en permanence à l'emplacement d'une des stations avant les travaux de réhabilitation.

Avant tout réaménagement du site, le résultat obtenu montre une augmentation très faible de l'exposition due au radon 222 , sauf à proximité immédiate du dépôt où la moyenne annuelle dépasse la LAI de $24 \%$ en $1990,5 \%$ en 1991 pour l'isotope 222 (station 2) et $26 \%$ en 1992 pour l'isotope 220 (station 3) ; cependant, ce dépassement ne pourrait concerner qu'une personne fictive séjournant en permanence en périphérie de ce terrain, ce qui constitue une hypothèse discutable.

Fin 1992, les travaux de réhabilitation du site ont été entrepris. Dès le mois de mai 1993, soit après la phase de terrassement et de compactage du terrain, les niveaux d'EAP ${ }_{v}$ enregistrés sur les stations 2 et 3 sont du même ordre de grandeur que ceux observés en dehors de l'influence du site.

Les valeurs d'EAP d $_{\mathrm{v}}$ obtenues ainsi que les flux d'émission radon mesurés dans la phase finale de la réhabilitation du site montrent que les objectifs fixés par l'arrêté préfectoral sont atteints, valorisant ainsi les techniques de réaménagement mises en œuvre [5].

\section{RÉFÉRENCES}

[1] BERNHARD S., VAUZELLE Y., ZETTWOOG P. - Measurement of the radiological impact of environmental radon 222 releases from heaps of solid wastes from the mineral industry. IRPA International conference, Johannesburg, February 20-24, 1995 (à paraître).

[2] FRANCE - Décret $n^{\circ}$ 66-450 du 20 juin 1966 relatif aux principes généraux de protection contre les rayonnements ionisants (J.O. du 30 juin 1966) modifié par décret $\mathrm{n}^{\circ}$ 88-521 du 18 avril 1988, J.O.R.F., 6 mai 1988, 6264-6298).

[3] FRANCE - Décret $n^{\circ}$ 90-222 du 9 mars 1990 complétant le règlement général des industries extractives institué par le décret $\mathrm{n}^{\circ}$ 80-331 du 7 mai 1980, J.O.R.F., 13 mars 1996, 3067-3071.

[4] LABED V., WITSCHGER O., ROBÉ M.C., SANCHEZ B. - Radon 222 emission flux and soil atmosphere interface : comparative analysis of different measurements techniques. Radiat. Prot. Dosim., 1994, 56, (1/4) 271-273.

[5] LABED V., ROBÉ M.C., BENEITO A., MAUREL J.M., RICHON P. Rehabilitation of a uranium-ore processing residues storage site at Le Bouchet, Paris region, France. International symposium on the Natural radiation environnement (NRE VI), Montréal, June 5-9, 1995 (à paraître).

[6] ROBÉ M.C., RANNOU A., LE BRONEC J. - Radon measurement in the environment in France. Radiat. Prot. Dosim., 1992, 45, (1/4) 455-457. 